\title{
VARIAÇÕES GRANULOMÉTRICAS, MINERALÓGICAS E ALCALINAS EM RESÍDUOS INERTIZADOS DA HYDRO ALUNORTE
}

\author{
E. R. PINHEIRO ${ }^{*}$, O. J. C. FERNANDEZ, J. CH. COSTA, E. M. VALENTE, P. J. CARVALHO \\ Instituto Federal de Educação, Ciência e Tecnologia do Pará \\ raiolerika22@gmail.com*
}

Submetido 15/10/2017 - Aceito 14/11/2017

DOI: $10.15628 /$ holos.2017.6401

\section{RESUMO}

A lama vermelha (LV), resíduo da etapa de clarificação do processo Bayer, possui milhões de toneladas acumuladas em extensas áreas por muitos anos. Para melhor aproveitamento deste resíduo é importante conhecer suas propriedades físicas e mineralógicas em todas as camadas inertizadas. Neste estudo foram avaliadas as variações granulométricas, mineralógicas e alcalinas da lama vermelha da Hydro Alunorte, ao longo de suas camadas inertizadas em um perfil de até $2 \mathrm{~m}$ de profundidade por meio de técnicas de caracterização de materiais como: sedimentação, difração de Raios-X e análise de $\mathrm{pH}$. As análises mostram que o resíduo possui granulometria predominantemente na fração silte, ainda com frações menores de argila e areia. Apresentam hematita, goethita, gibbsita, sodalita e anatásio como fases majoritárias. O pH mostra a LV com
\end{abstract}

uma variação de $\mathrm{pH}$ de 10,8 a 11,7 fortemente alcalino. O perfil apresentou variações tanto para alcalinidade como para a granulometria, assim como de um ponto para outro na bacia de rejeitos. Mineralogicamente, o perfil das camadas inertizadas apresentou regularidade das fases predominantes. As variações de granulometria e $\mathrm{pH}$ foram influenciadas pela topografia da bacia, devido as variações altimétricas, erosões e deslizamentos provocados pelas chuvas e ventos. A alcalinidade e granulometria, analisadas no tempo atual, podem apresentar outros resultados em outro período de estudo. Enquanto que, a mineralogia presente ao longo do depósito e em profundidade, com os fenômenos citados acima permanecem potencializando a mesma.

PALAVRAS-CHAVE: lama vermelha, camadas inertizadas, granulometria, caracterização.

\section{GRANULOMETRIC, MINERALOGIC AND ALKALINE VARIATIONS IN INERTIZED WASTE FROM HYDRO ALUNORTE}

\begin{abstract}
Red mud (RM), the residue from the clarification stage of the Bayer process, has milions of tons accumulated over extensive areas for many years. For better use of this residue it is important to know its physical and mineralogical properties in all the inert layers. In this study, the granulometric, mineralogical and alkaline variations of the red mud of the Hydro Alunorte were evaluated along their inert layers in a profile up to $2 \mathrm{~m}$ deep by means of characterization of materials such as: sedimentation, X-ray diffraction and $\mathrm{pH}$ analysis. The analyzes show that the residue has granulometry predominantly in the silt fraction, still with smaller fractions of clay and sand. Hematite, goethite, gibbsite, sodalite and anatase are the majorities phases. The $\mathrm{pH}$
\end{abstract}

of the RM range of 10.8 to 11.7 strongly alkaline. The profile presented variations for both alkalinity and granulometry, as well as from one point to another in the basin. Mineralogically, the profile of the inertized layers presented regularity of the predominant phases. The granulometry and $\mathrm{pH}$ variations were influenced by the topography of the basin, due to the altimetric variations, erosions and landslides caused by rainfall and wind. The alkalinity and granulometry, analyzed at the actual time may present others results in another period of study. While the mineralogy is present along the deposit and in depth, with the phenomena mentioned above remain potentiating the same.

KEYWORDS: red mud, granulometry, inert layer, characterization. 


\section{INTRODUÇÃO}

A lama vermelha (LV) é um resíduo sólido obtido através do processo Bayer. No Pará, as principais empresas envolvidas na extração do minério de alumínio são as seguintes: MRN Mineração Rio do Norte, ALCOA, e a Hydro Paragominas (MP). Villar (2002) classificou a granulometria da lama vermelha, na faixa dos siltes que varia de $10 \mu \mathrm{m}(0,01 \mathrm{~mm})$ a $100 \mu \mathrm{m}$ $(0,1 \mathrm{~mm})$.

As LVs apresentam como característica principal uma elevada alcalinidade que podem variar o pH de 10 a 13 (Hind, 1999). Segundo Rivas et al. (2010), as mesmas podem atingir até 13,6. De acordo com a norma NBR 10.004/2004, da Associação Brasileira de normas Técnicas (ABNT), a LV é classificada como resíduos Classe I - perigoso (alta corrosividade e reatividade), de tal forma que sua manipulação inadequada pode acarretar uma série de problemas ambientais. Segundo Hildebrando et al. (2006), os minerais presentes na lama vermelha do norte do Brasil possuem em sua constituição elementos da rocha de origem como: a gibbsita, hematita, goethita, anatásio, quartzo e uma pequena quantidade do argilomineral caulinita. Esse estudo contribuirá no conhecimento de variações prováveis de fases e tamanhos de grãos em profundidade.

Este trabalho tem como objetivo analisar as propriedades físico-químicas, tamanhos de grãos e mineralogia da lama vermelha em diferentes profundidades do Depósito de Rejeitos Sólidos (DRS) da empresa Hydro-Alunorte localizada no município de Barcarena no Estado do Pará. Através de ensaios de caracterização de materiais que permitam a identificação do $\mathrm{pH}$, caracterização de fases mineralógicas e a distribuição granulométrica, considerando o tempo de deposição e a profundidade das camadas inertizadas.

\section{REVISÃO BIBLIOGRÁFICA}

Segundo o Sampaio et al. (2005) e Antoniassi (2010), a bauxita é composta pelo principal constituinte de valor econômico gibbsita $\mathrm{Al}(\mathrm{OH}) 3$ e dificilmente para climas tropicais, boehmita $\gamma$ $\mathrm{AlO}(\mathrm{OH})$ e/ou diásporo $\alpha-\mathrm{AlO}(\mathrm{OH})$, por apresentar em sua composição química o alumínio. E suas principais impurezas são: caulinita $\mathrm{Al}_{2} \mathrm{Si}_{2} \mathrm{O} 5(\mathrm{OH}) 4$, óxi-hidroxidos de ferro hematita $\mathrm{Fe}_{2} \mathrm{O}_{3}$ e goethita $\mathrm{FeO}(\mathrm{OH})$, óxido de titânio TiO2 (rutilo e anatásio, ambos com mesma estrutura cristalina tetragonal). De acordo com Kotschoubey et al. (2005), as bauxitas são rochas formadas por lixiviação intensa de rochas subaéreas e altas temperaturas no Pará, fazendo com que seus teores de ferro e/ou alumínio sejam elevados e diminuam os teores de silício.

A bauxita produzida pela Mineração Rio do Norte é transportada por $1.000 \mathrm{~km}$ ao longo dos rios Trombetas e Amazonas e desembarcada no porto de Vila do Conde, a da mineradora Hydro Paragominas, por mineroduto por cerca de $244 \mathrm{~km}$. Essa bauxita é conduzida à Hydro Alunorte para a produção de alumina. A alumina abastece a Albrás (900 mil toneladas/ano), também é exportada para outras indústrias de alumínio, no Brasil e no exterior (Braga, 2010).

O rejeito da produção de alumina é a lama vermelha, e de acordo com Erdócia (2011), a lama vermelha é obtida durante o processo Bayer como produto rejeito do tratamento hidrometalúrgico da bauxita, para a obtenção de alumina. O processo consiste das seguintes operações: digestão, clarificação, evaporação, precipitação e calcinação. Em cada etapa podendo influenciar nas características mineralógicas desse material. De acordo com Sampaio et al. (2005), a digestão consiste em dissolver o hidróxido de alumínio da bauxita com a utilização de 
soda cáustica $(\mathrm{NaOH})$. O hidróxido de alumínio se apresenta em maior proporção nos seguintes minerais da bauxita: gibbsita, boehmita e diásporo. E seu contato com a soda cáustica forma uma solução supersaturada de aluminato de sódio (Na2O.Al2O3). Então, Hind et al. (1999) e Silva Filho et al. (2007), mostram que a lama vermelha é o resíduo insolúvel da etapa de clarificação do processo Bayer, composta por óxidos insolúveis de ferro, quartzo, aluminossilicatos de sódio, carbonatos, aluminatos de cálcio e dióxido de titânio.

Os fatores que influenciam na sedimentação das partículas de lama vermelha e consequentemente na granulometria são: floculantes ou defloculantes, composição mineralógica, micromorfologia e a forma de como o resíduo da bauxita é despejado no DRS. Antunes et al. (2011) analisou a granulometria da lama vermelha de uma refinaria de bauxita da cidade de Alumínio do interior de São Paulo, usando o método da pipeta, mostrando uma possível classificação no tamanho médio do diâmetro de suas partículas, com cerca a 57 - $64 \%$ os quais correspondem à faixa de $0,05 \mathrm{~mm}$ a $0,002 \mathrm{~mm}$ de diâmetro, ou seja, fração silte. Com essas dimensões, a LV é classificada como um material arenoso-argilo-silitosa, o qual é caracterizado por apresentar coesão mesmo a seco, porosidade muita pequena, intensos fenômenos de capilaridade, alguma plasticidade e existência de fenômenos de adsorção.

A lama vermelha possui mineralogia que depende do tipo de bauxita processada. Antoniassi (2010) mostra que a bauxita com características gibbsiticas predominam em ambientes tropicais (por exemplo Brasil, Suriname, Índia entre outros), e a bauxita com feições boehimiticas diaspóricas predominam em ambientes temperados, como na Europa e China. Assim, as fases minerais mais comuns da LV do Brasil são a hematita $\mathrm{Fe} 2 \mathrm{O} 3$, a goethita $\mathrm{FeO}(\mathrm{OH})$, a magnetita $\mathrm{Fe} 3 \mathrm{O} 4$, a boehmita $\gamma-\mathrm{AlO}(\mathrm{OH})$, o quartzo $\mathrm{SiO}_{2}$, a sodalita $\mathrm{Na} 4 \mathrm{Al} 3 \mathrm{Si} 3 \mathrm{O} 12 \mathrm{Cl}$ e a gipsita $\mathrm{CaSO}_{4} 2 \mathrm{H} 2 \mathrm{O}$, com menor presença de calcita $\mathrm{CaCO}_{3}$ e gibbsita $\mathrm{Al}(\mathrm{OH}) 3$ (Brunori et al., 2005; Pradhan et al., 1996 \& Yalçin et al., 2000).

Para $\circ \mathrm{pH}$, as pesquisas existentes a respeito de caracterização de lama vermelha geralmente relatam que a LV não é tóxica, porém a ABNT - NBR 10.004/2004 da Associação Brasileira de Normas Técnicas, citada anteriormente neste trabalho, a classifica como resíduo Classe I - perigoso (alta corrosividade e reatividade).

\section{METODOLOGIA}

\subsection{Materiais}

Para a realização deste trabalho utilizou-se amostras do P1 (ponto 1) e P5 (ponto 5) coletadas por Costa (2015) da área de depósito de rejeito sólidos (DRS) de LV da empresa Hydro Alunorte, localizada no município de Barcarena - Pará, cerca de $120 \mathrm{Km}$ de Belém. Foram coletadas 5 amostras de 8 pontos de diferentes profundidades de $40 \mathrm{~cm}, 80 \mathrm{~cm}, 120 \mathrm{~cm}, 160 \mathrm{~cm}$ e $200 \mathrm{~cm}$, totalizando 40 amostras no total (Figura 1). 


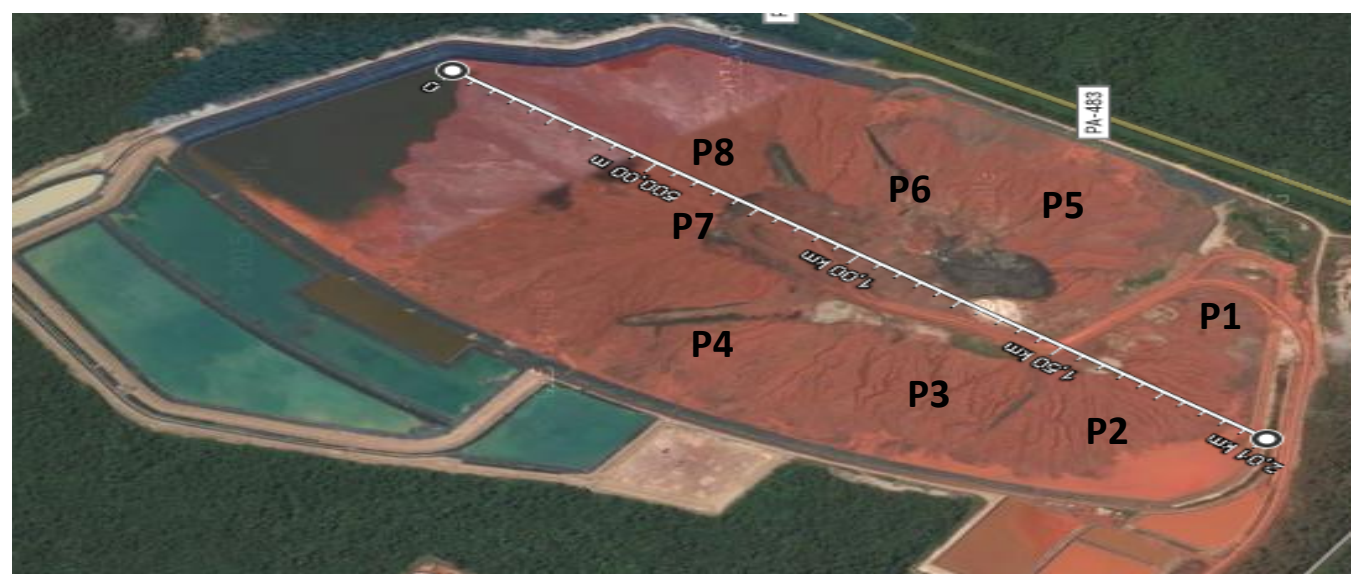

Figura 1: Depósito de rejeito sólidos da Hydro Alunorte - PA.

Para esse estudo, as amostras foram escolhidas de modo que estas fossem comparadas entre si. Sendo que as amostras do ponto 1 corresponde ao período de deposição mais antigo, de 1996 a 2000. E o ponto 5, ao período de 2006 a 2008. De modo a avaliar as mudanças mineralógicas de um período para o outro.

\subsection{Métodos}

\subsubsection{Preparação das amostras}

As amostras in natura (P1 e P5) passaram pelos seguintes processos respectivamente: secagem ( 30 minutos a $60{ }^{\circ} \mathrm{C}$ ) com o objetivo de retirar a umidade, moagem e peneiramento (200 mesh), como pode ser observado na Figura 2.


Figura 2: Preparação das amostras.

As amostras utilizadas por Costa (2015), de P1 à P8 passaram por uma preparação, que partiu do seguinte procedimento: filtragem à vácuo por 15 minutos (Figura 2A), filtragem à quente a $95{ }^{\circ} \mathrm{C}$, ambas com o objetivo de eliminar o teor cáustico. E secagem por 8 horas em estufa de $110{ }^{\circ} \mathrm{C}$ para eliminação da umidade, (Figura 2B). E cominuição manual, e posterior peneiramento na malha de 60\#, (Figura 2C).

\subsubsection{Análise do $\mathrm{pH}$}

Para a medição do $\mathrm{pH}$ das amostras foi executado o seguinte método: $50 \mathrm{ml}$ de água destilada e 2 gramas de lama vermelha em pó foram previamente secadas e moídas; logo foi realizado sua mistura com um bastão de vidro; a seguir se mediu o pH através de um pHmêtro padrão com faixa 0.0 a 14 , resolução de temperatura 0.5 @ C/ㅇF e exatidão $\pm 0.2 \mathrm{pH}$, previamente 
calibrado. Foram usadas tanto as amostras de P1 e P5 in natura, como amostras P1 e P5 tratadas por filtrações.

\subsubsection{Análise granulométrica}

A análise granulométrica foi realizada por sedimentação de sólidos em meio líquido utilizando-se o método da pipeta (Camargo et al., 2009). Para assim, verificar a frações granulométricas do material.

O procedimento partiu do seguinte método (Figura 3). Em uma proveta de $1000 \mathrm{ml}$ (dividida em 7 partes de 5 em $5 \mathrm{~cm}$ ) foi colocado água destilada e $21 \mathrm{~g}$ de amostra in natura (P1 e P5) de cada camada previamente fragmentadas, secadas a $60{ }^{\circ} \mathrm{C}$ por 1 hora e peneiradas na malha de 200\#. A proveta com a mistura foi agitada, com o movimento da boca para o fundo e com uma mangueira foi tirado cada altura. Depois de esperar o tempo determinado para sedimentar com o auxílio da equação da Lei de Stokes. As amostras foram colocadas na estufa a $60{ }^{\circ} \mathrm{C}$ até secar totalmente a água. Depois foram pesadas e levadas para análises de difração de raios-X.

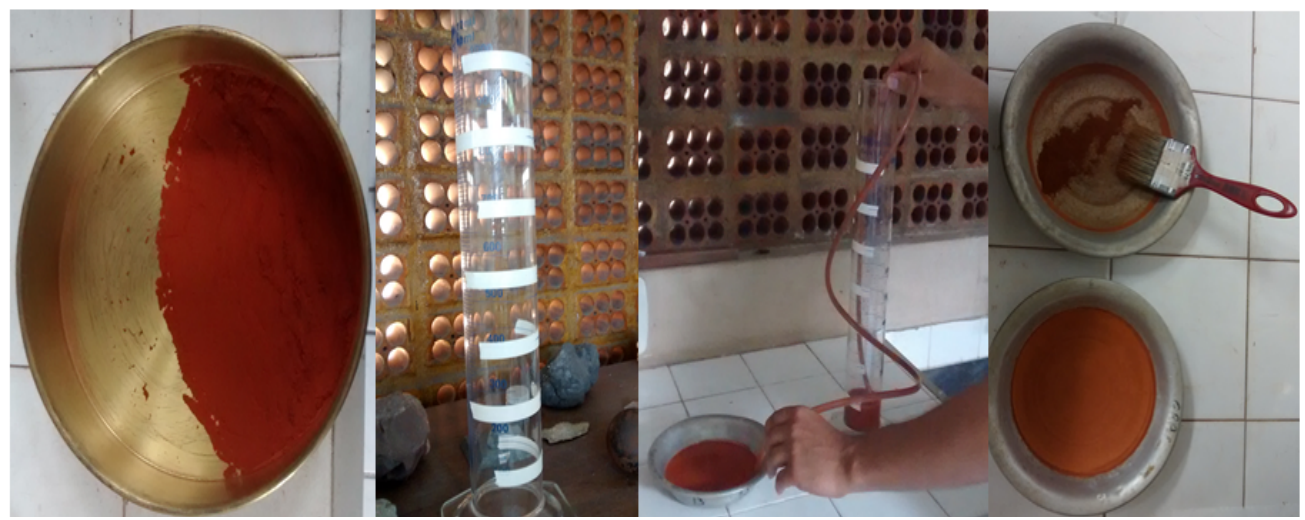

Figura 3: Preparação da amostra para sedimentação de LV.

Os diâmetros das partículas (Dp) foram definidos de acordo com as pesquisas realizadas, pois verifica que a lama vermelha está na faixa granulométrica dos siltes, que varia de $2 \mu \mathrm{m}$ até $60 \mu \mathrm{m}$, assim para determinar a velocidade e consequentemente o tempo de sedimentação através dos seguintes diâmetros de partículas (Dp) pré-determinado: 1, 3, 5, 10, 2038 e 74 m.

\subsubsection{Análise por difração de Raios-X (DRX)}

Para as análises por DRX foi utilizado o difratômetro D2 Phaser da Bruker e interpretadas no programa X'Pert HighScore Plus. Essas análises serviram para identificação de fases mineralógicas presentes nas amostras de LV do P1 e P5.

\section{RESULTADOS E DISCUSSÃO}

4.1. $\mathrm{pH}$

As amostras in natura apresentaram uma faixa de variação de pH entre 10,6 a 11,7. Depois de passar pela etapa de preparação, a amostra apresentou uma variação de pH entre 10,6 a 11 (tabela 1). Nota -se que o rejeito possui característica alcalina e seu pH diminuiu pouco depois da preparação da amostra por filtragens. Através das análises foi possível obter os seguintes resultados: 
Tabela 1: pH das amostras in natura do ponto 1 e ponto 5.

\begin{tabular}{c|ccc|c}
\hline \multirow{2}{*}{ Amostra } & \multicolumn{2}{|c|}{ In natura } & \multicolumn{2}{c}{ Tratadas por filtrações } \\
\cline { 2 - 5 } & Ponto 1 & Ponto 5 & Ponto 1 & Ponto 5 \\
\hline A1 & 11.7 & 11.1 & 10.8 & 10.8 \\
\hline A2 & 11.5 & 11.7 & 11.0 & 10.6 \\
\hline A3 & 11.4 & 10.8 & 10.8 & 10.6 \\
\hline A4 & 11.1 & 11.0 & 11.0 & 10.8 \\
\hline A5 & 11.0 & 10.6 & 10.6 & 10.6 \\
\hline
\end{tabular}

Esses resultados mostram um pH com forte alcalinidade, como indicado por Hind (1999), que pode atingir ate 13 ou mais segundo outros autores.

\subsection{Granulometria}

Através das pesquisas bibliográficas obtidas neste estudo, foram analisados que as lamas vermelhas apresentavam granulometria na fração silte $(\varnothing 0,002 \mathrm{~mm}$ e $0,06 \mathrm{~mm}$ ) e argila ( $\varnothing<$ $0,002 \mathrm{~mm}$ ). E de acordo com a norma NBR 7181/88 para solos siltosos e argilosos recomenda utilizar a sedimentação para a análise granulométrica, a fim de verificar se havia material maior que $0,074 \mathrm{~mm}$, foi utilizada a peneira de abertura de $200 \#(0,074 \mathrm{~mm})$, tendo como resultado nenhum material retido na peneira, tão somente houve material passante, assim não foi necessário utilizar peneiras com malhas menores a 200\# e seguir para o processo de sedimentação de acordo com a norma. Na tabelas 2 e 3 se mostram esses resultados e na figura 4 as curvas granulométricas.

Tabela 2: Diâmetro em função da massa do P1.

\begin{tabular}{c|cccccc}
\hline \multirow{2}{*}{$\begin{array}{c}\text { Diâmetro } \\
\text { (mm) }\end{array}$} & \multicolumn{5}{|c}{ Fração granulométrica passante (\%) } \\
\cline { 2 - 6 } & P1A1 & P1A2 & P1A3 & P1A4 & P1A5 \\
\hline 0,074 & 100,00 & 100,00 & 100,00 & 100,00 & 100,00 \\
\hline 0,038 & 47,88 & 46,53 & 29,04 & 61,53 & 65,07 \\
\hline 0,02 & 37,25 & 39,29 & 24,96 & 50,39 & 54,20 \\
\hline 0,01 & 29,26 & 30,73 & 20,84 & 41,15 & 44,27 \\
\hline 0,005 & 23,58 & 22,89 & 16,81 & 30,01 & 34,58 \\
\hline 0,003 & 15,70 & 15,04 & 12,28 & 20,58 & 24,56 \\
\hline 0,001 & 7,99 & 8,15 & 8,20 & 11,14 & 14,28 \\
\hline
\end{tabular}

Tabela 3: Diâmetro em função da massa do P5.

\begin{tabular}{c|c|c|c|c|c}
\hline \multirow{2}{*}{$\begin{array}{c}\text { Diâmetro } \\
\text { (mm) }\end{array}$} & \multicolumn{5}{|c}{ Fração granulométrica passante (\%) } \\
\cline { 2 - 6 } & P5A1 & P5A2 & P5A3 & P5A4 & P5A5 \\
\hline 0,074 & 100,00 & 100,00 & 100,00 & 100,00 & 100,00 \\
\hline 0,038 & 61,15 & 72,08 & 57,96 & 68,87 & 65,53 \\
\hline 0,02 & 51,67 & 59,99 & 48,04 & 57,30 & 55,14 \\
\hline 0,01 & 41,89 & 49,60 & 38,22 & 46,16 & 44,86 \\
\hline 0,005 & 33,28 & 38,04 & 29,19 & 35,82 & 33,82 \\
\hline 0,003 & 22,87 & 26,16 & 20,57 & 24,31 & 22,46 \\
\hline 0,001 & 12,62 & 13,80 & 13,16 & 12,85 & 11,74 \\
\hline
\end{tabular}


As análises granulométricas no ponto P1 e P5 (Figura 4), com diâmetro de corte d80, mostram que as frações apresentam pouca variabilidade no diâmetro das partículas, todas elas tendendo para a fração silte, entretanto se considerarmos o d50, nitidamente observam-se, no geral, diâmetros de grão variados, todas elas na fração silte, com d20 os diâmetros tendem fração silte - argila. Ao comparar as amostras dos pontos P1 com P5 (tabelas 2 e 3 ) observamos que no diâmetro de 0,038 mm (P5) ocorrem porcentagens passantes maiores (acima de 0,038 $\mathrm{mm}$ ), enquanto que a proporção passante no ponto 1 a maior parte do material é menor que $0,038 \mathrm{~mm}$. Em geral em ambos pontos, a fração silte é predominante.

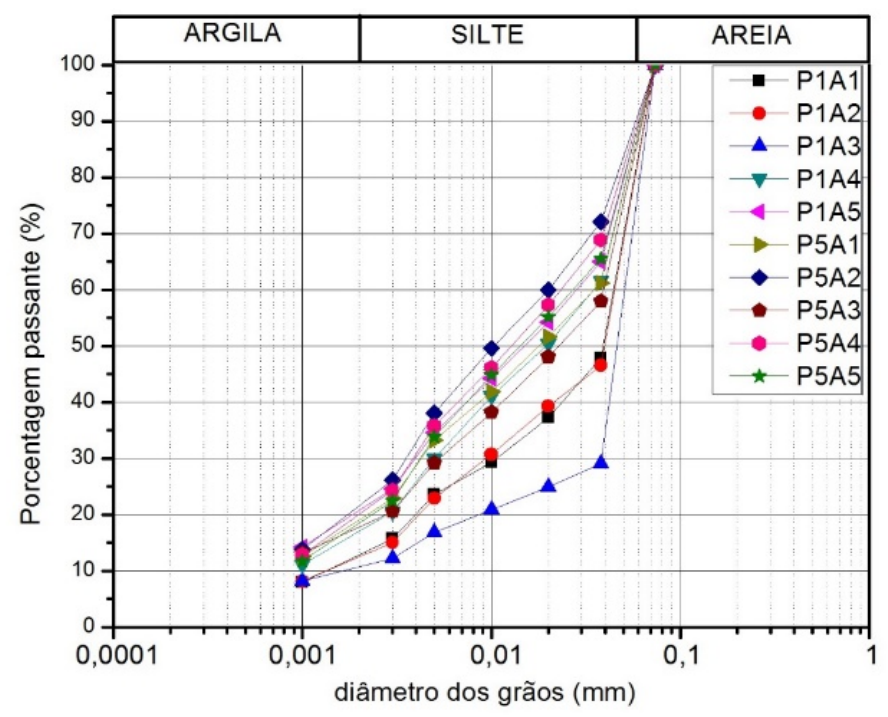

Figura 4: Granulometria das amostras estudadas.

\subsection{Difração de Raios-X}

De acordo com as figuras de 5 a 6 as fases predominantes no P1 e P5 amostrados são: hematita (PDF: 01-079-1741), sodalita (PDF: 01-070-5029), goethita (PDF: 01-081-0463), gibbsita (PDF: 00-033-0018) e anatásio (PDF: 01-084-1285). Por vezes foram identificados bayerita (PDF: 00-008-0096), calcita (PDF: 01-072-1652), quartzo (PDF: 01-085-0798) e cancrinita (PDF: 01-0898074). Em geral, em profundidade as fases não apresentam grandes variações, apenas no quantitativo. Dessas fases, sodalita e cancrinita, sugerem sejam fases neoformadas durante a operação metalúrgica de lixiviação. Essas fases tipo zeolíticas, sugerem transição de sodalita para cancrinita, e estão relacionadas a quantidade de $\mathrm{NaOH}$ e aumento de temperatura (Santos et al. 2014). É notoria também a presença de gibbsita, essa fase já foi notada em LVs nos estudos de Antoniassi (2010); Brunori et al.(2005); Pradhan et al.(1996); Yalçin \& Sevinç (2000). 


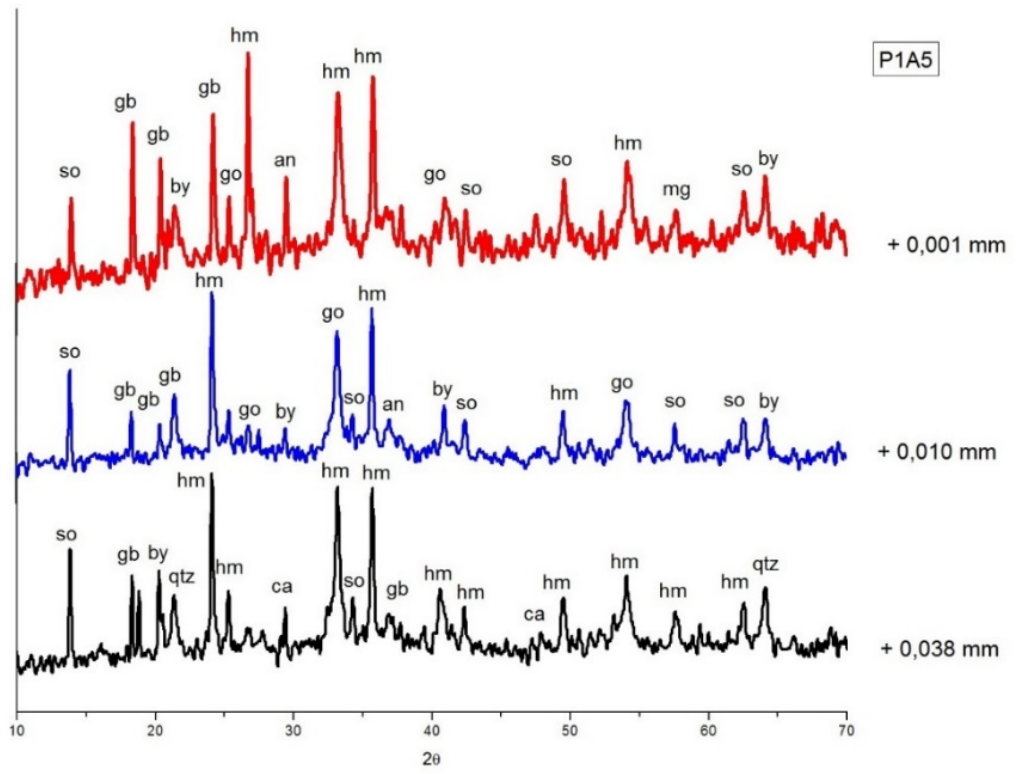

Figura 5: Difratogramas de Raios- $X$ das amostras de lama vermelha do ponto P1A5. gb=gibbsita, $h m=h e m a t i t a$, so=sodalita, go=goethita, by=bayerita, an= anatásio, ca=calcita, qtz=quartzo.



Figura 6: Difratogramas de Raios-X das amostras de lama vermelha do ponto P5A5. gb=gibbsita, hm=hematita, so=sodalita, go=goethita, an=anatásio, by=bayerita, ca=calcita, qtz=quartzo.

\section{CONCLUSÕES}

A lama vermelha da Hydro Alunorte apresentou variações físico-químicas, granulométricas e mineralógicas ao longo de suas camadas inertizadas no tempo. Em sua forma natural, a LV do DRS apresentou uma variação no $\mathrm{pH}$ de 10,6 a 11,7, apesar de ser lavada e filtrada para ser colocada na área de depósito, este resíduo ainda é considerado alcalino. A granulometria classificada da lama vermelha se encontra na faixa de 0,0001 $\mathrm{mm}$ a 0,1 $\mathrm{mm}$ de diâmetro de tamanho de grão, com predominância da fração silte: para quantidades maiores (cerca a 90\%) estão na fração silte-argila e para quantidades menores (10\%) estão na fração areia. As camadas inertizadas mais profundas concentram as frações silte (com tendência para areia). 
As camadas inertizadas com o tempo de deposição de resíduo, são compostas principalmente de hematita, sodalita, goethita, gibsita e anatásio, e minoritariamente calcita, quartzo e cancrinita. Essas fases estão determinadas, principalmente, pela procedência da bauxita. As fases mineralógicas influenciam na granulometria das partículas de LV ao longo do perfil inertizado. Quantidade maiores de hematita, gibbsita, sodalita e goethita apresentam granulometria de $+0,010 \mathrm{~mm}$ a $+0,038 \mathrm{~mm}$ dentro da fração silte.

No presente trabalho pode-se perceber que não há uma regularidade de granulometria e teor alcalino da lama ao longo do depósito (DRS) da Hydro Alunorte, estando fortemente influenciada pela procedência da bauxita. A granulometria corresponde à fração silte, $\mathrm{pH}$ extremamente alcalino e fase mineralógica predominante de hematita. Essas variações de granulometria e $\mathrm{pH}$ também são influenciadas pela topografia do DRS, devido as variações altimétricas, erosões e deslizamentos provocados pelas chuvas e ventos. A alcalinidade e granulometria, analisadas no tempo atual, podem apresentar outros resultados em outro período de estudo. Enquanto que a mineralogia presente ao longo do depósito e em profundidade, com esses fenômenos permanecem potencializando a mesma.

\section{REFERÊNCIAS}

Antoniassi. J. L. (2010). A difração de raios x com o método de Rietveld aplicada à bauxitas de porto de trombetas. PA. Dissertação de mestrado. USP, São Paulo.

Antunes, M.L.P., Da Conceição, F.T., \& Navarro, G.R.B. (2011). Caracterização da Lama Vermelha Brasileira (Resíduo do Refino da Bauxita) e Avaliação de suas Propriedades para Futuras Aplicações. $3^{\text {rd }}$ International Workshop Advances in Cleaner Production, São Paulo.

Braga, R. M. Q. L. (2010). A utilização de uma camada de solo compactado como revestimento impermeabilizante de fundo de bacias de disposição de lama vermelha produzida em Barcarena-PA. Tese de doutorado. Universidade Federal do Pará. Instituto de Geociências, Belém - PA.

Brunori, C., Cremisini, C., Massanisso, P., Pinto, V., Torricelli, L. (2005). Reuse of a treated red mud bauxite waste: studies on environmental compatibility, Journal of Hazardous Materials, v. 117, n.1, pp. 55-63.

Camargo, O.A; Moniz, A.C; Jorge, J.A; Valadares, J.M.A.S. (2009). Métodos de análise química, mineralógica e física de solos do Instituto Agronômico de Campinas. Campinas, Instituto Agronômico. 77 p. (Boletim técnico, 106, edição revisada e atualizada).

Costa, J. C. (2015). Variações químico mineralógicas em camadas inertizadas de lama vermelha da Hydro Alunorte. Trabalho de conclusão de curso. IFPA, Belém.

Erdócia, F. A. B. (2011). Difração de Raios X em Minerais de Bauxita e Análise através de Refinamento pelo Método de Rietveld. Dissertação de Mestrado (pg. 1 - 2), UFPA. Belém.

Hildebrando, E. A.; Souza, J.A.S; Neves, R.F. (2006). Influência do tipo de argila nas propriedades físicos-mecânicas de corpos cerâmicos obtidos com lama do processo Bayer. CBECIMAT Congresso Brasileiro de Engenharia e Ciência dos Materiais, Foz do Iguaçu. 
Hind, A. R., Bhargava, S. K., \& Grocott, S. C. (1999). The surface chemistry of Bayer process solids: a review Colloids and Surfaces A: Physicochemical and Engineering Aspects. 359-374.

Kotschoubey, B., Calaf, J.M. C., Lobato, A. C.C., Leite, A. S., \& Azevedo., C. H. D. (2005). Caracterização e gênese dos depósitos de bauxita da província bauxifera de Paragominas, noroeste da bacia do Grajaú, nordeste do Pará/oeste do Maranhão. In: Onildo Marini, Emanuel Teixeira Queiroz e Benedicto Waldir Ramos. (Org) Caracterização de Depósitos Minerais em Distritos mineiros da Amazônia. 1ed. Brasília: DNPM - CT/MINERAL - ADIMB, v. 1, p. $691-782$.

NBR 10.004/2004. ABNT. Associação Brasileira de Normas Técnicas.

NBR 7181/88. ABNT. Associação Brasileira de Normas Técnicas.

Pradhan, J., Das, S. N., Das, J., Rao, S. B., \& Thakur, R.S. (1996). Characterization of Indian red muds and recovery of their metal values", Light Metals, pp. 87-92.

Rivas, J.M., Galdino, L.G., Vasconcelos, N.S.L.S., Paiva, A.E.M., Cabral, A.A., \& Angélica, R.S. (2010). Estudo do comportamento térmico e propriedades físico-mecânicas da lama vermelha. vol. $15 n^{\circ 3}$, pp. 445 - 460. Rio de Janeiro: Revista Matéria.

Sampaio, J. A.; Andrade, M. C. \& Dutra, A. J. B. (2005). Comunicação Técnica elaborada para Edição do Livro Rochas \& Minerais Industriais: Usos e Especificações. Pág. 279 a 304. CETEM, Rio de Janeiro.

Santos, C.G.M; Passos, F.A.C.M; Silva; A. A. S.; Barbato, C;N; Sampaio, J.A.;.Garrido, F. M. S; Silva, F. A. N. G . Estudo de Síntese e Transição entre as Fases Zeolíticas Sodalita e Cancrinita. HOLOS, Ano 30, Vol. 3 - Edição Especial - XXV ENTMME / VII MSHNT.

Silva filho, E. B., Alves, M. C. \& Da Motta, M. (2007). Lama Vermelha da indústria de beneficiamento de alumina: produção, características, disposição e aplicações alternativas. São Paulo.

Villar, L. F de S. (2002). Estudo do adensamento e ressecamento de resíduos de mineração e processamento de bauxita. 443 p. (Doutorado em Engenharia Civil) - Pontifícia Universidade Católica do Rio de Janeiro, Rio de Janeiro.

Yalçin, N., Sevinç, V. (2000). Utilization of bauxite waste in ceramic glazes, Ceramics International, n 26, pp. 485-493. 